\title{
Introduction to Numerical Experiment on Plasma Focus using Lee Model Code
}

\author{
P. Gautam* and R. Khanal \\ Central Department of Physics, Tribhuvan University, Kirtipur, Kathmandu, Nepal \\ *Corresponding author: om.gautam49501@gmail.com
}

\begin{abstract}
A dense plasma focus is a table top machine producing a short-lived very hot plasma and can cause nuclear fusion. Lee Model Code is a computer simulation package, which was successfully used in Mather and Fillipov type plasma focus. Lee Model couples the electrical circuit with the plasma focus dynamics, radiation and thermodynamics to simplify the complicated dynamics of plasma focus. This package enables us to simulate and analyze all of the gross properties. In this paper we present the importance of current fitting and the relation of it with other plasma focus parameter and overview of Lee Model Code together with physical basis, scope and the results obtained from the Lee Model Code.
\end{abstract}

Keywords: Plasma dynamics, Nuclear fusion, Plasma focus device, Lee Model Code,

\section{INTRODUCTION TO PLASMA FOCUS DEVICE}

The dense plasma focus (DPF) is a coaxial gun, with the inner electrode, the anode, is electrically insulated from the outer electrode, cathode. After achieving a high vacuum condition, desired gas is admitted at a pressure of a few millibar or torr. The plasma is originated when capacitor bank is discharged through a low inductance transmission line (as spark or rail gap switches are closed) with in very short interval of rise time in the range of ns [1]. The DPF produces abundant multi-radiation, a wide spectrum of photons and particles and is the subject of many studies and applications [2]. The plasma focus is divided into two sections: The first section is pre-pinch (axial) section and the second one is the radial pinch phase. The main function of the axial phase is to delay the pinch until the capacitor discharge approaches to maximum

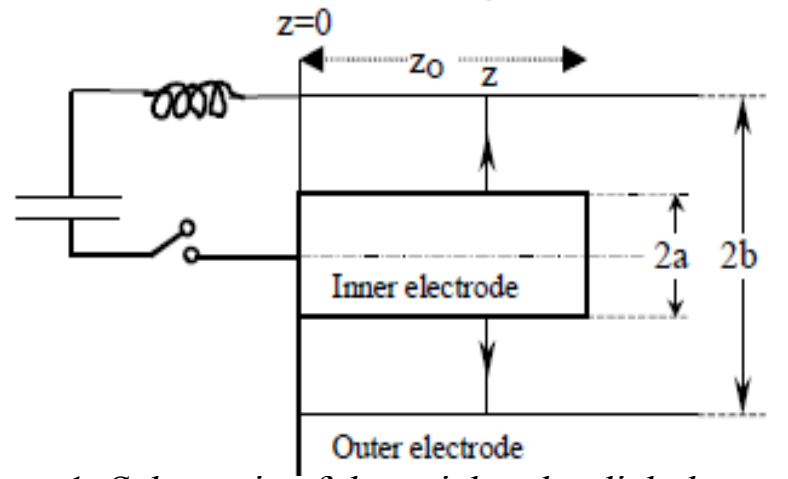

discharge current; which is done by driving a current sheet down an axial (acceleration) section until the capacitor current approaches its peak value. After which the pinch current sheet is allowed to undergo transition into a radial phase. That's why the pinch starts and occurs at the top of the current sheet [3]. The two phase mechanism of the plasma focus [1] is shown in Figure 1. An insulating backwall separates the inner electrode from the outer concentric cathode. The electrode system is enclosed in a chamber, evacuated and typically filled with gas at about $1 / 100$ of atmospheric pressure. Once the capacitor voltage switches onto the focus tube, the break down occurs axis-symmetrically between the cathode and anode across the back-wall. The current sheet lifts off the back-wall as the magnetic field $\left(\mathrm{B}_{\theta}\right)$ and the inducing current $\left(\mathrm{J}_{\mathrm{r}}\right)$ rises to a sufficient value [2].

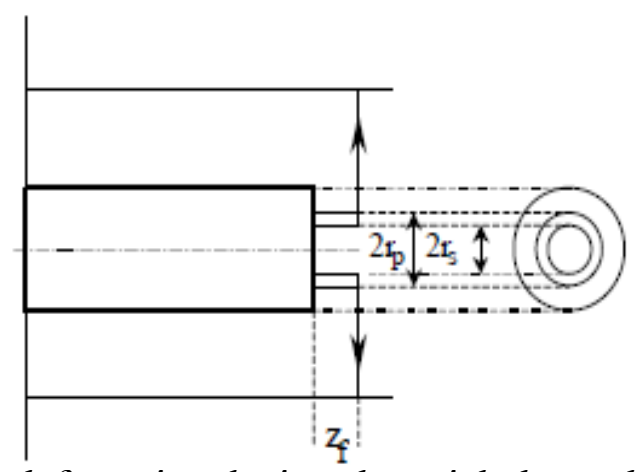

Figure 1: Schematic of the axial and radial phases. The left section depicts the axial phase, the right section the radial phase. In the left section, $z$ is the effective position of the current sheath-shock front structure. In the right section $r_{s}$ is the position of the inward moving shock front driven by the piston at position $r_{p}$. Between $r_{s}$ and $r_{p}$ is the radially imploding slug, elongating with a length $z_{f}$ [4] 
Axial Phase: When $B_{\theta}$ and $J_{r}$ rise sufficiently the Lorentz force $\left(\mathrm{J}_{\mathrm{r}} \times \mathrm{B}_{\theta}\right)$ pushes the current sheet and then accelerated supersonically into the tube which is similar to the mechanism of linear motor. When the discharge reaches to its quarter the current sheet reaches to the end of the axial section; which is possible because of the reason of matching of the length of the tube, speed of the current sheet and the rise time of the capacitor discharge. The axial phase typically lasts 1-3 $\mu$ sec for a plasma focus of several $\mathrm{kJ}$ [3].

Radial Phase: Cylinder of current is formed by slipping off the current sheet to the end of the anode electrode, after which it is pinched inwards. The collapsing (imploding) plasma cylinder has two boundaries; the inner face of the wall is collapsing shock front with radius $r_{s}$ and outer face of the wall is collapsing magnetic plasma piston (current sheet) with radius $r_{p}$. There is annular (ring shaped) layer in between the shock front and the magnetic piston. The imploding shock front with higher and higher speed coalesces on axis resulting in a super-dense and super-hot plasma column pinched onto the axis (Figure 2); which last typically for short time (in the range of ns) for a small plasma focus. Then the column breaks up and explodes [3].

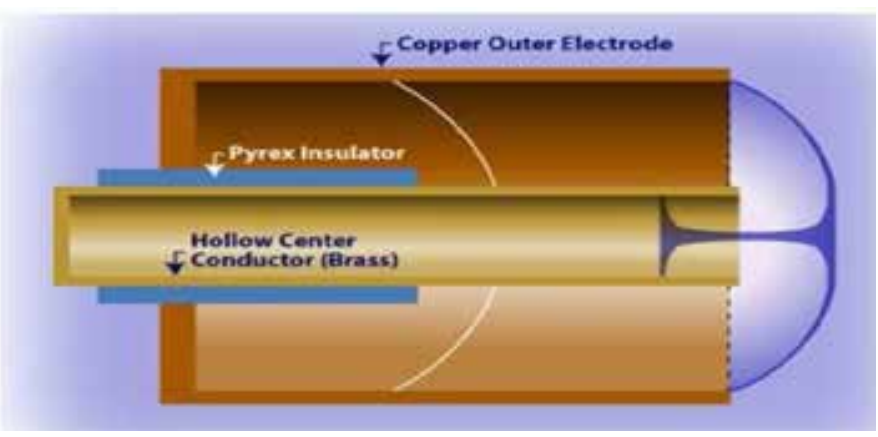

Figure 2: Dense plasma focus device

Figure 3 shows typical shadowgraphs taken of the actual radially imploding current sheet-shock front structure [3, 4]. The shadowgraph are taken in sequence, at different time. The indication in the shadowgraphs are relative to the moment viewed to be the moment of maximum compression. The moment of the maximum compression is taken as $\mathrm{t}=0$. The quality of plasma compression can be clearly seen to be very good, with excellent axissymmetry and a very well compressed dense phase.
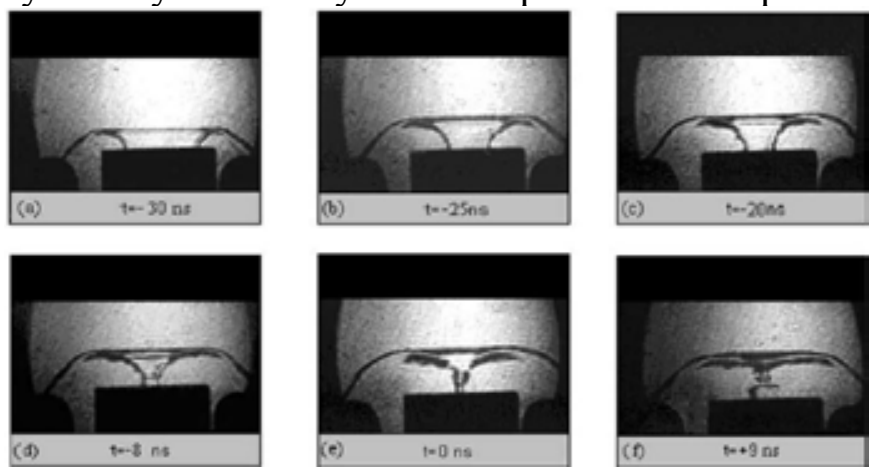

Figure 3: Shadowgraphic sequence showing formulation of the plasma focus pinch. Sequential images from (a) to (d) show the plasma column being 'pinched' radially inwards; (e) being time of maximum compression forming the hot and dense 'fusion' plasma.

\section{THE LEE MODEL CODE}

The Lee model code couples the electrical circuit with plasma focus, thermodynamics and radiation, enabling realistic simulation of all gross focus properties [5]. The basic model described in 1984 [2], was successfully used to assist several experiments [6]. The radiation-coupled dynamics was included in 5-phase code, which is successful to lead numerical experiments on radiation cooling [7]. Reflected shock and radiative phase are added to the earlier model to simulate the X-ray emission from the plasma focus [8]. The signal-delay slug was incorporated together with real gas thermodynamics and radiation-yield terms, which is so crucial to radial simulation and assisted other research projects [9-11 ] and web published in 2000 [12] and 2005 [13]. All subsequent versions of Lee code are improved versions and signal delay slug is incorporated as a must have feature. Plasma self- absorption was included [12] in 2007, improving soft X-ray yield simulation. The code has been used extensively as a complementary facility in several machines, such as; UNU/ICTP PFF [6, 10, 14], NX2 [9, 11], NX1 [11]. Information obtained includes axial and radial dynamics [14], soft X-ray (SXR) emission characteristics and yield $[10,11]$, design of machines 
$[6,10,11,14]$, optimization of machines $[10,11$, 14] together with the adaptation of the Filippovtype [15]. Plasma focus SXR yield calculations [16], pinch current and SXR yield limitations [17], optimization of SXR yield [16,17], radiative collapse and cooling [18], line radiation [19], current stepped PF [20], PF neutron yield calculations [21], current and neutron yield limitations [22], neutron saturation [23] and extraction of diagnostic data [24] and the anomalous resistance phase(RAN) data [25] from the current signals have been studied applying the code [5]. The inclusion of the neutron yield, $Y_{n}$, using beam target mechanism [21] is one the great step in the development, incorporated in the versions [5] of the code (later than RADPF5.13), resulting in realistic $Y_{n}$ scaling with $I_{\text {pinch }}$ [21]. The Lee code can be downloaded freely from [5]. A brief description of the 5- phase Lee model code is given in the following sub-sections.

\subsection{Axial phase}

This phase is described by a snowplow model with an equation of motion coupled to a circuit equation. The equation of the motion includes the axial phase model parameters and simply known as mass sweptup and current factors and noted by the symbol $\mathrm{f}_{\mathrm{m}}$ and $f_{c}$ respectively. $f_{m}$ is responsible for porosity of the current sheet, inclination of the moving current sheet shock front structure, boundary layer effects and other unspecified effects which creates effects on the amount of mass in the moving, during the axial phase and $f_{c}$ is responsible for fraction of the current effectively driving the structure, during the axial phase. This phase is shown at the left part of Figure 1.

\subsection{Radial inward shock phase}

This phase is described by four coupled equations using an elongating slug phase. The first equation calculates the radial inward shock speed from the driving magnetic pressure. The second equation calculates the axial elongation speed of the column. The third one calculates the speed of the current sheath and the fourth equation is the speed of the current sheath and the fourth equation is the speed of the current sheath and the fourth equation is the circuit equation. As the model parameter $\mathrm{f}_{\mathrm{mr}}$ and $\mathrm{f}_{\mathrm{cr}}$ presents as radial mass swept-up and current factor respectively and incorporates for all of the three radial phases. The model parameter $f_{\text {mr }}$ is responsible for the effects on the amount of mass in the moving slug during the radial phase and $f_{c r}$ is responsible for the fraction of the current effectively driving the radial slug. This phase is shown in the right part of Figure 1 and also in Figure $4[4,5]$.

\subsection{Radial Reflected Shock (RS) phase}

This phase is also described by the four coupled equations, these being for the reflected shock moving radially outwards, the piston moving radially inwards, the elongation of the annular column and the circuit. The same model parameters $f_{m r}$ and $f_{c r}$ are used as in the radial inward shock phase. The plasma temperature behind the reflected shock undergoes a jump by a factor close to 2. Number densities are also computed using the reflected shock jump equations. This phase is clearly shown in Figure 4.

\subsection{Slow compression (Quiescent) or pinch phase}

This phase is described by three coupled equations, these being the piston radial motion, the pinch column elongation and circuit equation with the same model parameters as in the previous two phases. When the out-going reflected shock hits the in-coming piston the compression enters into the radiative phase. This phase is responsible for the emission of the radiation, neutron, ion beam and electron. The time of transit of the small disturbances across the pinched plasma column is set as the duration of the slow compression phase.

\subsection{Expanded Column Phase}

In this final phase again the Snow Plow model is used which means again two coupled equations are applied similar to the axial phase. This phase is not so important as it occurs after the focus pinch. 


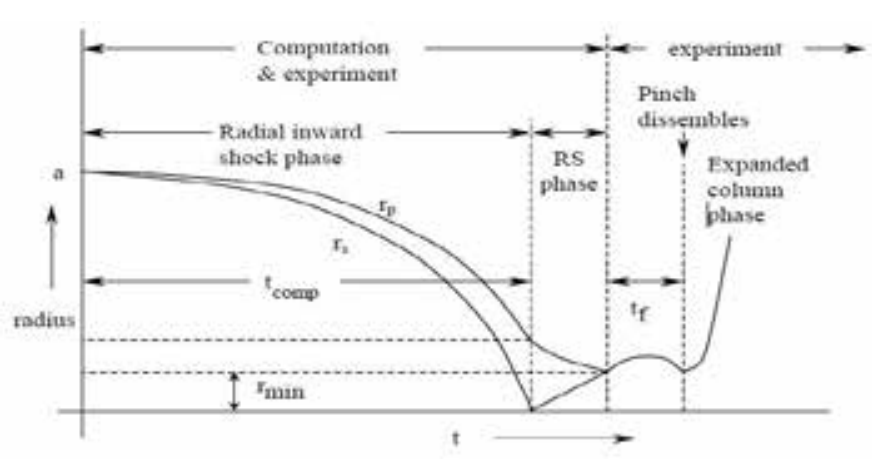

Figure 4: Schematic of radius versus time trajectories to illustrate the radial inward shock phase when $r_{s}$ moves radially inwards, the reflected shock (RS) phase when the reflected shock moves radially outwards, until it hits the incoming piston $r_{p}$ leading to the start of the pinch phase $\left(t_{f}\right)$ and finally the expanded column phase.

\section{NUMERICAL EXPERIMENT PROCEDURE}

The Lee Model Code is configured to work as any plasma focus by providing the appropriate tube parameters $\mathrm{b}$, a, and $\mathrm{z}_{0}$; the bank parameters, $\mathrm{L}_{0}, \mathrm{C}_{0}$ and the stray resistance $r_{0}$ and operational parameters $\mathrm{V}_{0}$ and $\mathrm{P}_{0}$ and the gas fill. The tube parameter of the device shows the size of tube used in the plasma focus device, bank parameters shows the capacity of the inductor, capacitance and the resistance used in the combination of circuit of device and operational parameter are operating voltage and the pressure of gas used there. The standard practice to fit the total discharge current waveform to experimentally measured value is done by adjusting the four model parameters axial mass swept-up factor $\left(\mathrm{f}_{\mathrm{m}}\right)$, axial current factor $\left(f_{c}\right)$, radial mass swept-up factor $\left(f_{m r}\right)$ and radial plasma current factor $\left(f_{\mathrm{cr}}\right)[5]$. The current trace is the best indicator of the gross performance of the plasma focus device. Important information like axial and the radial phase dynamics and the essential energy transfer are quickly visible from the current trace, which shows the importance of the fitting of the current trace.

For the fitting of total discharge current with measured discharge first of all the measured data for total discharge current is either taken from direct laboratory experiments or picked out from published article. Then the computed total current waveform is fitted with the measured total discharge waveform by varying the model parameters $f_{m}, f_{c}, f_{\text {mr }}$ and $f_{c r}$ one by one until the computed waveform fits well with the measured waveform. As a first step of fitting the axial factors $f_{m}, f_{c}$ are adjusted until the rising slope of the total trace, rounding off of the peak current and the peak current itself are in reasonable fit with the measured total current trace. Then fitting for the radial model parameters $f_{m r}, f_{c r}$ is carried until the computed slope and the depth of the dip agree with the measured values. A typical fitted trace is shown in Figure 5. Once the current trace matches the numerical experiment can be conducted by varying any physical parameter of interest.

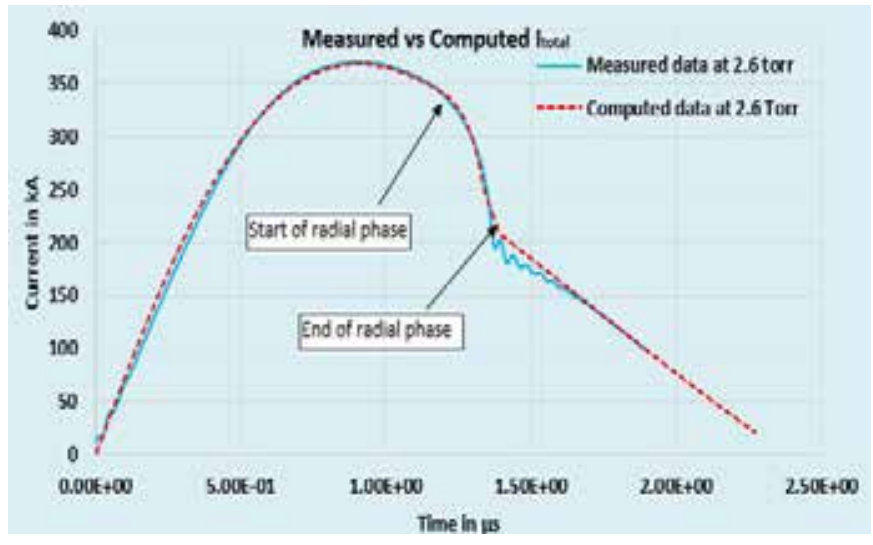

Figure 5: The fitting of computed current trace to the measured current trace

\section{CONCLUSION}

It is well known that the current trace of the focus is the best indicator of the gross performance of the plasma focus. The axial and radial phase dynamics and the crucial energy transfer into focus pinch are among the important information that is quickly apparent from the current trace. The exact time profile of the total current trace is governed by the bank parameters, the operational parameters, focus tube geometry together with the mass swept-up and the fraction of sheath current and the variation of these fractions through the axial and radial phases. Once the current trace is matched, the fitted model parameters assure that the computation proceeds with all physical mechanisms accounted for typically for that plasma focus device. All the plasma dynamics can then be obtained after each run of the code. 
Using the Lee model code, series of experiments have been systematically carried out to observe the behavior patterns of the plasma focus. Some of these experiments include:

- pinch current limitation effect as static inductance is reduced $[17,22]$;

- $\quad$ neutron and SXR scaling laws [21,23, 27];

- a global scaling law for neutrons versus storage energy combining experimental and numerical experimental data [4];

- the nature and cause of neutron saturation [4];

- compression enhancement by circuit manipulation and radiative cooling and collapse $[18,20]$

- experiments on PF1000 neutron yield [28] and

- comparison of measured neutron yield versus pressure curves for different machines against computed results [29].

\section{REFERENCES}

[1] F. Castillo, J. J. E. Herrera, J. Rangel, A. Alfaro, M. A. Maza and V. Sakaguchi, Brazilian Journal of Physics 32, 1 (2002)

[2] S. Lee; Radiations in Plasmas II (Ed. B. McNamara), World Scientific, Singapore, 978, (1984)

[3] S. Lee, Twelve years of UNU/ICTP PFF-a review, Abdus Salam ICTP, Trieste, Italy, http://eprints.ictp.it/31/ (1998)

[4] S. Lee and S. H. Saw, Numerical Experiments Workshop (Research) on Plasma Focus manual, Dhulikhel, Nepal (21 September 2014)

[5] S Lee; Radiative Dense Plasma Focus Computation Package: RADPF

http://www.intimal.edu.my/school/fas/ UFLF/File1RADPF.htm;

http://www.plasmafocus.net/IPFS/modelpackage/ File1RADPF.html (archival websites)

[6] S. Lee and A. Serban, IEEE Trans Plasma Science 24, 1101, (1996)

[7] J B Ali, Development and studies of a small plasma focus, PhD Thesis, Universiti Teknologi Malaysia, Malaysia (1990)

[8] M. H. Liu and S. Lee; ICPP and EPS Conf. on Contr. Fusion and Plasma Physics, ECA, 22C, 2169 (1998)

[9] S. Bing; 2000 Plasma dynamics and X-ray emission of the plasma focus, PhD Thesis, NIE, Singapore
[10] M.H. Liu, X.P. Feng, S.V. Springham and S. Lee, IEEE Trans.Plasma Sci. 26, 135 (1998)

[11] S. Lee, P. Lee, G. Zhang, X. Feng, V.A. Gribkov, M. Liu, A. Serban and T. Wong, IEEE Trans. Plasma Sci. 26, 1119 (1998)

[12] S. Lee; 2000/2007 http://ckplee.myplace.nie. edu.sg/plasmaphysics/

[13] S. Lee; 2005 ICTP Open Access Archive: http://eprints.ictp.it/85/

[14] S. Lee et al, American J. Phys. 56, 62 (1988)

[15] V. Siahpoush, M. A. Tafreshi, S. Sobhanian and S. Khorram, Plasma Phys. Control. Fusion 47, 1065 (2005)

[16] M. Akel, Sh. Al-Hawat, S.H. Saw and S. Lee, J. Fusion Energ 29, 223 (2010)

[17] M. Akel, Sh. Al-Hawat and S. Lee, J. Fusion Energ. 29, 94 (2010)

[18] S. Lee, S.H. Saw and J. Ali, J. Fusion Energ. 32, 42 (2013)

[19] M. Akel and S. Lee; J. Fusion Energ. 32, 111 (2013)

[20] S. Lee and S. H. Saw, J. Fusion Energ. 31, 603 (2012)

[21] S. Lee and S. H. Saw, J. Fusion Energ. 27, 292 (2008)

[22] S. Lee and S. H. Saw, Appl. Phys. Lett. 92, 021503 (2008)

[23] S. Lee, Plasma Phys. Control. Fusion 50, 105005 (2008)

[24] S. Lee, S.H. Saw, P.C.K. Lee, R.S. Rawat and H. Schmidt, Appl. Phys. Lett. 92, 111501 (2008)

[25] S. H. Saw, R. S. Rawat, P. Lee, A. Talebitaher, A. E. Abdou, P. L. Chong, F. Roy, J. Ali, and S. Lee, IEEE Trans. on Plasma Science 93, 3813 (2013)

[26] A. Serban and S. Lee, Plasma Sour. Sci. Technol. 6, 78 (1997)

[27] S. Lee, R. S. Rawat, P. Lee and S. H. Saw, J. of App. Phy. 106, 023309 (2009)

[28] S. H. Saw, D. Subedi, R. Khanal, R. Shrestha, S. Dugu and S. Lee, J. Fusion Energ. 33, 684 (2014)

[29] S. H. Saw, P. Lee, R. S. Rawat, R. Verma, D. Subedi, R. Khanal, P. Gautam, R. Shrestha, A. Singh and S. Lee, J. Fusion Energ. submitted (2014) 